\title{
MANEJO ANESTÉSICO Y ANALGÉSICO DEL PACIENTE ORTOGERIÁTRICO
}

\author{
Gaspar Ramírez B. ${ }^{1}$, Dr. Pablo Miranda H. ${ }^{2}$ y Fernando Altermatt C. ${ }^{3}$
}

Key words: Conduction anesthesia, Geriatrics, Perioperative Period.

\section{INTRODUCCIÓN}

La Organización Mundial de la Salud define al adulto mayor (AM) como toda persona mayor de 60 años. Para nadie es un misterio que la población a nivel mundial está envejeciendo. El porcentaje de adultos mayores, está creciendo a razón de $1 \%$ anual, con un aumento esperado de 287 millones el año 2013 a 417 millones el 2050'. En Chile, se espera que la población de AM crezca de 2.553.352 (estimada a junio del año 2014), a 3.207.729 de personas para el año 2020, constituyendo un $17 \%$ de la población nacional y acumulando un aumento del $171 \%$ con respecto al año $1990^{2}$.

El avance tecnológico, las técnicas quirúrgicas y las unidades de cuidados intensivos, han hecho posible un aumento en intervenciones quirúrgicas en esta población. El grupo mayor a 65 años posee una mayor tasa anual de procedimientos con anestesia y se espera que más de la mitad de los mayores de 65 años necesiten al menos una intervención quirúrgica durante el resto de su vida ${ }^{3,4}$.

En la cirugía traumatológica en particular, se espera que aumente el número de intervenciones en este grupo etario. En un estudio epidemiológico desarrollado en Japón, más de la mitad de los pacientes operados de cualquier causa ortopédicatraumatológica fueron mayores de 65 años ${ }^{5}$.

El AM es una población que posee gran cantidad de comorbilidades: un tercio de ellos tienen tres o más enfermedades preexistentes y un $80 \%$ presenta al menos una comorbilidad ${ }^{3}$. Esto cobra especial importancia cuando se sabe que las enfer- medades subyacentes, junto a la duración y la urgencia de la cirugía son los principales factores de riesgo para una intervención quirúrgica, por sobre la edad cronológica ${ }^{3}$.

Lo mismo se replica para cirugías ortopédicas, como la artroplastía total de rodilla y de cadera ${ }^{6,7}, o$ también para la cirugía por fractura de cadera, donde se ha demostrado que no es la cirugía en sí, sino que son las comorbilidades perioperatorias las que determinan la recuperación del estado funcional ${ }^{8}$.

Es de vital importancia para el equipo médico y en especial para el anestesiólogo conocer y manejar de forma adecuada el dolor perioperatorio asociado a una cirugía traumatológica en el AM, no sólo por la alta prevalencia de esta población, sino porque el manejo inadecuado del dolor puede asociarse a complicaciones relevantes que impactan el resultado final.

\section{CAMBIOS FISIOLÓGICOS DEL ADULTO MAYOR Y SU RELACIÓN CON LA ANESTESIA}

El envejecimiento es un proceso fisiológico normal, común a todas las personas, que se manifiesta con cambios irreversibles y la mayoría de las veces progresivos, que afecta el funcionamiento de los órganos, disminuyendo así la reserva de estos y su capacidad de mantener la homeostasis lo que puede aumentar la sensibilidad a los diferentes estresores $^{3}$.

Estos cambios fisiológicos deben ser conocidos

\footnotetext{
División de Anestesiología, Facultad de Medicina Pontificia Universidad Católica de Chile.

Interno de Medicina.

Residente Anestesia Regional.

Profesor Asociado, División de Anestesiología.
} 
Tabla 1. Dirección y magnitud aproximada de los cambios fisiológicos en el adulto mayor y su efecto en variables farmacocinéticas. Modificado de Macintyre et $\mathrm{al}^{9}$.

\begin{tabular}{|c|c|c|c|}
\hline $\begin{array}{l}\text { Variable fisiológica } \\
\text { General }\end{array}$ & Cambio & Consecuencia cinética/dinámica & Estrategia farmacológica \\
\hline $\begin{array}{l}\text { Gasto cardiaco } \\
\uparrow \mathrm{RVS}, \uparrow \mathrm{PA} \rightarrow \mathrm{HVI} \rightarrow \text { Disfunción } \\
\text { diastólica } \rightarrow \text { Dilatación } \\
\text { auricular } \rightarrow \uparrow \mathrm{FA}\end{array}$ & $\downarrow 0-20 \%$ & $\begin{array}{l}\downarrow \text { volumen compartimiento central } \\
\uparrow \text { concentración máxima después } \\
\text { de un bolo }\end{array}$ & $\begin{array}{l}\text { Disminuir dosis del bolo inicial } \\
\text { Disminuir velocidad de inyec- } \\
\text { ción }\end{array}$ \\
\hline Grasa & $\begin{array}{l}\uparrow 10-50 \%, \\
\text { luego } \downarrow\end{array}$ & $\begin{array}{l}\text { Cambios en el volumen de distri- } \\
\text { bución de ciertas drogas }\end{array}$ & $\begin{array}{l}\text { Dosis según peso real o peso } \\
\text { magro }\end{array}$ \\
\hline Masa muscular/ flujo sanguíneo & $\downarrow 20 \%$ & & \\
\hline Volumen plasmático & Cambios leves & & \\
\hline Agua corporal total & $\downarrow 10 \%$ & $\begin{array}{l}\downarrow \text { volumen de distribución (dro- } \\
\text { gas hidrosolubles) }\end{array}$ & \\
\hline Albúmina plasmática & $\downarrow 20 \%$ & $\uparrow$ fracción libre de la droga & $\begin{array}{l}\text { Cambios potenciales en el clea- } \\
\text { rance y la biodisponibilidad oral }\end{array}$ \\
\hline Alfa 1 glicoproteína & $\uparrow 30-50 \%$ & $\begin{array}{l}\leftrightarrow \text { clearance hepático de drogas } \\
\text { de alta extracción }\end{array}$ & $\begin{array}{l}\text { Cambios potenciales en los } \\
\text { efectos a nivel cerebral }\end{array}$ \\
\hline Unión a proteínas & Según droga & $\begin{array}{l}\uparrow \text { clearance hepático de drogas } \\
\text { de baja extracción } \\
\uparrow \text { captación cerebral de la droga }\end{array}$ & \\
\hline \multicolumn{4}{|l|}{ Hígado } \\
\hline Tamaño hepático & $\downarrow 25-40 \%$ & $\begin{array}{l}\downarrow \text { clearance hepático de drogas } \\
\text { de alta extracción }\end{array}$ & $\begin{array}{l}\text { Efecto mínimo en la dosis de } \\
\text { bolo endovenoso inicial }\end{array}$ \\
\hline Flujo sanguíneo hepático & $\downarrow 25-40 \%$ & $\begin{array}{l}\leftrightarrow \text { clerance hepático de drogas } \\
\text { de baja extracción }\end{array}$ & $\downarrow$ dosis de mantención \\
\hline Fase I (ej, oxidación) & $\downarrow 25 \%$ & $\begin{array}{l}\downarrow \text { clearance hepático (para al- } \\
\text { gunas drogas de baja extracción }\end{array}$ & $\begin{array}{l}\text { Cambios potenciales en la bio- } \\
\text { disponibilidad oral }\end{array}$ \\
\hline Fase II & Cambios leves & & \\
\hline \multicolumn{4}{|l|}{ Riñón } \\
\hline Cantidad de nefronas & $\downarrow 30 \%$ & $\downarrow$ clearance de drogas (polares) & $\begin{array}{l}\downarrow \text { dosis de mantención (de dro- } \\
\text { gas con metabolismo renal) }\end{array}$ \\
\hline Flujo sanguíneo renal & $\begin{array}{l}\downarrow 10 \% \text { por } \\
\text { década }\end{array}$ & $\begin{array}{l}\text { Leve efecto en el metabolismo } \\
\text { de opioides }\end{array}$ & $\begin{array}{l}\text { Asumir y monitorizar la acumu- } \\
\text { lación acelerada de metabolitos } \\
\text { polares activos (M6G) o tóxicos } \\
\text { (M3G, norpetidina) }\end{array}$ \\
\hline Flujo plasmático a los 80 años & $\downarrow 50 \%$ & $\begin{array}{l}\downarrow \text { clearance de metabolitos acti- } \\
\operatorname{vos}(\text { ej. M6G) }\end{array}$ & \\
\hline Tasa de filtración glomerular & $\downarrow 30-50 \%$ & & \\
\hline Clearance de creatinina & $\downarrow 50-70 \%$ & & \\
\hline \multicolumn{4}{|l|}{ Sistema Nervioso Central (SNC) } \\
\hline $\begin{array}{l}\text { Flujo sanguíneo y metabolismo } \\
\text { cerebral }\end{array}$ & $\downarrow 20 \%$ & $\downarrow$ distribución al SNC & Leve efecto neto en la dosis \\
\hline Masa encefálica & $\downarrow 20 \%$ & $\begin{array}{l}\downarrow \text { aparente del volumen de distri- } \\
\text { bución cerebral }\end{array}$ & \\
\hline $\begin{array}{l}\text { Transporte activo de la barrera } \\
\text { hematoencefálica. (eflujo) }\end{array}$ & $\begin{array}{l}\downarrow \text { (según la } \\
\text { droga) }\end{array}$ & $\begin{array}{l}\uparrow \text { aparente del volumen de distri- } \\
\text { bución cerebral }\end{array}$ & $\begin{array}{l}\downarrow \text { dosis de bolo inicial durante } \\
\text { titulación }\end{array}$ \\
\hline Umbral del dolor & Leve variación & & Sin cambios al titular \\
\hline $\begin{array}{l}\text { Respuesta según concentración } \\
\text { plasmática (opioides). }\end{array}$ & $\begin{array}{l}\uparrow 50 \% \text { para al- } \\
\text { gunos opioides }\end{array}$ & $\uparrow$ respuesta a opioides & $\begin{array}{l}\downarrow \text { dosis de bolo durante titu- } \\
\text { lación } \\
\downarrow \text { dosis de mantención }\end{array}$ \\
\hline
\end{tabular}

RVS: resistencia vascular sistémica; PA: presión arterial; HVI: hipertrofia ventricular izquierda; FA: fibrilación auricular; M3G: morfina-3-glucorónido; M6G: morfina-6-glucorónido. 
y considerados en el momento de evaluar y manejar dolor, ya que van a provocar cambios farmacológicos relevantes a la hora de seleccionar una estrategia analgésica.

Los cambios fisiológicos más importantes y la conducta clínica sugerida, se indican en la Tabla 1. A continuación se describen otros cambios que se producen y su repercusión farmacológica y clínica.

En relación a la composición corporal, se produce una disminución del volumen del compartimiento central, aumentando la concentración plasmática de algunas drogas luego de un bolo ${ }^{10}$. Los medicamentos liposolubles aumentan su volumen de distribución, con la probabilidad de aumentar el tiempo de efecto clínico, mientras que los medicamentos hidrosolubles, lo ven disminuido ${ }^{11}$. Se produce una disminución de la cantidad de drogas ácidas unidas a proteínas, sin embargo, estos cambios no determinan diferencias clínicas y no son un factor predominante en la farmacocinética de estas drogas en el envejecimiento. Solo en pacientes con polifarmacia, estos cambios podrían favorecer la interacción entre drogas ${ }^{10,12}$. El nivel de alfa 1 glicoproteína ácida plasmática, que transporta drogas básicas, parece aumentar, pero tampoco existen repercusiones clínicas relevantes en este caso ${ }^{10,12}$.

A nivel de sistema cardiovascular, existe una disminución de la contractilidad, un aumento del tiempo de contracción y rigidez miocárdica, aumento de la presión de llenado ventricular y una menor sensibilidad beta-adrenérgica ${ }^{13}$. En general, en los AM, el aumento del llenado telediastólico logra mantener el gasto cardiaco, pero se ha visto mujeres que pueden presentar una ligera disminución de este con el paso de los años ${ }^{10}$.

Debido a que el corazón del AM se hace más dependiente de volumen, pequeños cambios hemodinámicos pueden producir inestabilidad cardiovascular. Es por esto, que se debe tener una especial precaución con la administración de analgesia vía neuroaxial y su consecuente disminución de la respuesta simpática ${ }^{11}$.

En cuanto al sistema respiratorio, en el AM se afecta tanto desde el punto de vista estructural como funcional y aumenta el riesgo de desarrollar complicaciones respiratorias sin importar el tipo de anestesia o analgesia que se utilice ${ }^{14}$. Los centros respiratorios disminuyen su respuesta ventilatoria a la hipoxemia y a la hipercapnia. Además, existe un aumento de los efectos depresores de las benzodiacepinas, opioides y de los anestésicos no volátiles ${ }^{15}$. Por lo tanto, existe un mayor riesgo de desarrollar hipoxemia en la unidad de recuperación postanestésica por lo que se sugiere el uso de oxígeno en forma preventiva ${ }^{11}$.
Existe en el AM, además, una disminución de la distensibilidad pulmonar y de la caja torácica, un aumento del volumen residual y de la capacidad de cierre, elementos que provocan un aumento del espacio muerto anatómico ${ }^{11}$. El aumento de la capacidad de cierre por la edad, sumado a la disminución de la capacidad residual funcional con el uso de anestesia general, aumentan el riesgo de desarrollar atelectasias ${ }^{14}$. Por otro lado, el dolor postoperatorio y el uso de opioides sistémicos pueden contribuir a una reducción del volumen corriente y a una disminución del aclaramiento de secreciones en mayor medida en comparación a un paciente joven ${ }^{16}$.

En relación al sistema nervioso central, en el AM hay una menor respuesta simpática dada por una disminución significativa de la síntesis de neurotransmisores ${ }^{16}$. Existe un aumento de la actividad adrenérgica en forma basal, respondiendo al estrés con aumentos de la concentración de noradrenalina mayor que en las personas más jóvenes ${ }^{3}$. Sin embargo, este cambio no siempre tiene una importancia clínica, dado que se produce una disminución de la afinidad de la noradrenalina por sus agonis$\operatorname{tas}^{17} \mathrm{y}$ a que existe una menor respuesta autonómica dada por una menor sensibilidad de los barorreceptores $^{3,11}$. Estos cambios producen una disminución en la recuperación de la estabilidad ante situaciones de estrés hemodinámico, como también una mayor probabilidad de producir hipotensión con el uso de técnicas anestésicas neuroaxiales.

Se produce, por mecanismos no absolutamente claros, una disminución global de la reserva funcional cerebral, lo que conlleva a un aumento en la sensibilidad a los medicamentos anestésicos, una mayor incidencia de delirium perioperatorio y de disfunción cognitiva postoperatoria ${ }^{10}$.

Los cambios a nivel hepático, producen alteraciones farmacocinéticas en los medicamentos con alta extracción en este órgano, lo que tiene poco efecto en una dosis única inicial sin embargo, se pueden acumular medicamentos cuando se utilizan como infusión continua ${ }^{11}$.

El tracto gastrointestinal, en general, mantiene su funcionalidad, pero presenta a nivel gástrico una menor secreción de prostaglandinas y de bicarbonato, por lo que se deben utilizar con precaución los antiinflamatorios no esteroidales (AINEs) por su mayor probabilidad de producir daño en la mucosa gástrica en el $\mathrm{AM}^{16}$.

A nivel renal, a pesar de que el aclaramiento de creatinina disminuye, la creatinina plasmática tiende a permanecer estable dado la disminución de la masa muscular en el AM. Esto tiene especial relevancia debido a que se pueden acumular metabolitos activos o tóxicos de drogas con eliminación 
renal. Además, se altera la capacidad de conservar sodio, lo que sumado a una respuesta de sed disminuida, favorecen el riesgo de deshidratación ${ }^{10}$.

En cuanto a la temperatura corporal, toda anestesia produce una reducción de la temperatura central, especialmente por redistribución hacia la periferia. Los AM se vuelven más hipotérmicos durante la cirugía y demoran más tiempo en recuperar su temperatura en el postoperatorio al compararlo con pacientes jóvenes. Al respecto, se recomienda utilizar medidas que tiendan a disminuir la hipotermia y medir la temperatura en los AM sometidos a anestesia general con duración mayor a $30 \mathrm{~min}$ y anestesia neuroaxial durante cirugías de mayor duración ${ }^{18}$.

\section{IMPLICANCIAS PARA LA ANESTESIA REGIONAL}

\section{Anestesia peridural}

En el AM, el posicionamiento del paciente y la inserción de la aguja pueden ser más difíciles, dado que a pesar de que puede ser más fácil localizar estructuras óseas superficiales, la calcificación de estructuras ligamentosas pueden dificultar la entrada del trocar al espacio peridural o intratecal ${ }^{19}$. Los cambios estructurales y funcionales a nivel de columna y médula espinal se acompañan de cambios en la absorción, distribución y la duración de anestésicos locales y otros fármacos administrados. Existe una mayor propagación cefálica del anestésico local luego de inyección peridural, lo que implica que menores volúmenes son necesarios para cubrir el mismo número de dermátomos en comparación con pacientes más jóvenes ${ }^{20,21}$. La causa de este fenómeno es por una disminución del espacio peridural debido al cierre progresivo de los agujeros intervertebrales ${ }^{22}$.

En el AM, hay un inicio de acción más rápido tras la inyección peridural de anestésico local, debido a un aumento en la permeabilidad de las meninges ${ }^{20,23}$. En estos pacientes, la latencia disminuye, y de igual forma se incrementa la intensidad del bloqueo motor luego de la inyección peridural de bupivacaína ${ }^{20}$. Por lo tanto, para limitar la extensión del bloqueo analgésico y simpático luego de la administración peridural, se recomienda reducir la dosis, aunque no existen dosis óptimas para el adulto mayor ${ }^{3}$.

En estos pacientes, se ha observado una mayor respuesta a opioides en comparación con pacientes jóvenes. Por vía peridural, proporcionan analgesia postoperatoria prolongada y se requieren dosis menores de morfina. Esto también se ha demostrado en estudios en contexto de cirugías traumatológicas ${ }^{24}$.
Se piensa que estos cambios se deban probablemente a concentraciones más elevadas de opioides en LCR en comparación con población más joven ${ }^{3}$.

Para el uso de analgesia epidural controlada por el paciente, se sugiere que la dosis de administración continua y en bolo se reduzcan hasta en $50 \%$ en ancianos ${ }^{3}$.

\section{Anestesia espinal}

Luego de una anestesia espinal, se ha visto en ancianos una prolongación de la duración del bloqueo, debido a una disminución del flujo sanguíneo subaracnoídeo y consecutivamente, una disminución de la absorción del anestésico local ${ }^{3}$. La altura máxima de la anestesia espinal, al usar bupivacaína isobárica, es marginal ${ }^{25}$, pero de todas formas, el bloqueo motor completo, así como una mayor duración de la analgesia con este tipo de anestesia en ancianos, la hacen una alternativa satisfactoria para procedimientos quirúrgicos de las extremidades inferiores ${ }^{3}$.

Por otro lado, al usar soluciones hiperbáricas, el nivel de analgesia se incrementa entre tres a cuatro segmentos hacia cefálico, en comparación con población más joven. Además, existe una disminución del tiempo hasta la aparición del bloqueo motor ${ }^{26}$. Se debe estar atento a posibles complicaciones de la administración intratecal de opioides, las que se presentan con mayor frecuencia en el AM, como son retención urinaria y depresión respiratoria ${ }^{3,11}$.

La incidencia de compromiso neurológico producto de complicaciones hemorrágicas, específicamente hematomas espinales (y más frecuentemente epidurales) asociadas con la anestesia neuroaxial aumentan, entre otras cosas, con la edad, lo que se debe probablemente a una mayor dificultad en la técnica, asociado a indicación concomitante de fármacos tromboprofilácticos, a un eventual estrechez del canal vertebral y secundario a técnicas continuas. Sin embargo, estas técnicas son ampliamente utilizadas en los AM con un muy bajo grado se complicaciones de este tipo ${ }^{27}$.

\section{Bloqueo de nervio periférico}

En cuanto a los bloqueos de nervio periférico, son pocos los estudios que se han llevado a cabo en el AM, a pesar de ser una técnica muy utilizada en este grupo etario, en especial para cirugía de extremidades inferiores. Algunos estudios muestran mayor duración del bloqueo sensitivo y motor en ancianos en comparación con pacientes más jóvenes3. Se debe tener precaución en los adultos mayores, ya que poseen una vasculatura más frágil de los nervios periféricos y muchas veces, existencia de neuropatía previa al procedimiento, que se pu- 
diera agravar o confundir con un efecto adverso de la técnica ${ }^{11}$. Por otro lado, los AM poseen mayor riesgo de intoxicación sistémica por anestésicos locales que la población joven, más aun si poseen alguna patología crónica que comprometa la farmacocinética de las drogas, por lo que hay que reducir la masa total del anestésico local inyectado.

\section{CONSIDERACIONES ESPECIALES SOBRE EL DOLOR EN EL ADULTO MAYOR}

En el AM se producen diversos cambios tanto en la función nociceptiva como en la percepción y manifestación clínica del dolor, que transforman su evaluación y manejo en un verdadero desafío para el equipo médico.

Un manejo adecuado del dolor en el AM es fundamental, el dolor postoperatorio aumenta la probabilidad de desarrollar eventos adversos ${ }^{28}$. El manejo adecuado del dolor puede disminuir la incidencia de isquemia miocárdica y de complicaciones pulmonares, promover la deambulación precoz, acelerar la recuperación, disminuir el tiempo de estadía hospitalaria y reducir los costos médicos ${ }^{29,28}$.

\section{Delirium}

Una de las complicaciones asociadas más relevantes en el adulto mayor es el delirium postoperatorio. La incidencia puede incluso exceder el $50 \%$ en cirugías como las cardiacas o las traumatológicas ${ }^{14}$. Aunque el desarrollo de este cuadro es multifactorial, el dolor postoperatorio mal manejado puede ser una de las causas contribuyentes más importantes, aumentando el riesgo en relación a una valoración más alta del dolor ${ }^{29}$.

El delirium postoperatorio se ha asociado a un aumento de la mortalidad ${ }^{30}$. Se asocia a una tardanza en el inicio de la deambulación, del alta hospitalaria, mayor probabilidad de disfunción cognitiva luego del alta, mayor incidencia de reingreso hospitalario y a una menor probabilidad de recuperación de la capacidad para realizar actividades básicas de la vida diaria ${ }^{8}$.

\section{Evaluación del dolor}

En el adulto mayor existe una degeneración de neuronas sensitivas y pérdida de mielina en las astas dorsales de la médula espinal, al igual que una diminución de la sustancia P y somatostatina ${ }^{31}$. Se piensa que la disminución de neuronas noradrenérgicas y serotoninérgicas, contribuyen a la disminución de los mecanismos inhibitorios descendentes del dolor, lo que explicaría que en esta población exista una menor tolerancia al dolor ${ }^{31}$. Por otro lado, existe una disminución de la percepción del dolor y de los síntomas asociados con el aumento de la edad ${ }^{29}$. Además, muchos estudios sobre los cambios del nivel de estímulo necesario para gatillar dolor en ancianos demuestran en su mayoría un aumento de este umbral, sin embargo, la mayoría de ellos son metodológicamente deficientes ${ }^{11}$. De todas maneras, en general, en los adultos mayores existe una reducción de la función nociceptiva de fibras A delta y $\mathrm{C}$, un retraso en la sensibilización central, una disminución en la sensibilidad a los estímulos dolorosos de baja intensidad y una recuperación más lenta a la hiperalgesia ${ }^{11,29,32}$.

Desde el punto de vista clínico, en el adulto mayor muchas veces es difícil evaluar adecuadamente el dolor. Algunas causas que explican este fenómeno son la creencia de que el dolor es parte del envejecimiento, el miedo a requerir más medicamentos, a perder independencia y a requerir más procedimientos o pruebas diagnósticas ${ }^{33,34}$.

Los pacientes con disfunción cognitiva tienen la misma probabilidad de sentir dolor agudo frente a distintos estímulos, que los pacientes sin ella. A pesar de esto, este grupo de pacientes tiende a reportar menos dolor en una proporción directa a la intensidad de su disfunción ${ }^{35}$. Algunas explicaciones a esto serían la disminución de la memoria, la incapacidad para reportar el dolor y la mayor prevalencia de alexitimia ${ }^{36}$ (incapacidad de darse cuenta y de verbalizar las propias emociones).

Un problema frecuente en estos pacientes es que muchas veces existe un subtratamiento del dolor ${ }^{11,31}$. Un estudio en que se evaluó el dolor y el manejo de este en pacientes ancianos con y sin disfunción cognitiva con fractura de cadera, mostró que el grupo con disfunción cognitiva recibió un tercio menos de morfina que el grupo control ${ }^{37}$.

En cuanto a la forma de evaluar el dolor, existen variadas escalas. En general, para medir el dolor agudo, las más utilizadas son las escalas unidimensionales. Dentro de estas, las escalas categóricas (que utilizan palabras para describir la magnitud del dolor) como por ejemplo la Escala de Descripción Verbal (EDV), tienen una buena correlación con escalas más utilizadas como la Escala Visual Análoga $(E V A)^{38}$. Estas escalas tienen la ventaja de ser aplicables a los adultos mayores, dado a que son fáciles y rápidas de implementar. Por otro lado, existen escalas numéricas, como la Escala Numérica Verbal (ENV) y la EVA, que tienen como ventaja el tener un mayor abanico de opciones, con lo que se reduce la imprecisión a la hora de la evaluación. Las escalas unidimensionales que han demostrado ser adecuadas para su uso en pacientes de mayor edad, son 
la ENV, la Escala Facial de Dolor (EFD), la EDV, la Escala de Rating Numérico (ERN) y EVA ${ }^{39}$.

La EDV es una escala unidimensional categórica, que utiliza 6 frases descriptivas, de las cuales el paciente debe elegir la que más se relacione con su percepción del dolor. Es recomendada para su uso en adultos mayores que tengan dificultad para entender y usar la evaluación del 0 al 10 de la ERN. Existe además una variación de la EDV, el Termómetro del dolor (Figura 1), que incorpora una imagen visual de un termómetro con una alineación vertical de las frases descriptivas; esta escala es preferible en pacientes con disfunción cognitiva moderada a severa y para aquellos con dificultad para el pensamiento abstracto ${ }^{40}$.

En una comparación de cinco escalas (EVA, ENV, EFD, EDV, ERN), todas ellas fueron efectivas para discriminar diferentes niveles de dolor en el adulto mayor, sin embargo la EDV fue la más sensible y confiable, y la que se consideró como la mejor opción en el adulto mayor ${ }^{42}$.

En los adultos mayores que no pueden comunicarse, existen otros tipos de mediciones para el dolor. En general se basan en observaciones que pueden hacerse a las respuestas autonómicas y de comportamiento asociadas a dolor. Por ejemplo, puede buscarse un aumento de la frecuencia cardiaca o respiratoria o la presencia de diaforesis. Sin embargo, la ausencia de estas respuestas no es sinónimo de ausencia de dolor y, además, pueden verse en cuadros concomitantes como ansiedad, hipovolemia o sangrado $^{40}$. Los cambios de comportamiento pueden ser la agitación, fruncimiento del ceño, muecas o emisión de sonidos, pero no necesariamente son buenos marcadores en estos pacientes y son difíciles de interpretar ${ }^{31}$. Al respecto, existen más de 20 tipos de mediciones estandarizadas. Una de ellas es la lista de verificación de Indicadores No Verbales de Dolor, la que incluye 6 comportamientos relacionados con dolor (vocalizaciones, muecas, agitación, quejas verbales, aferramiento a objetos, masajes sobre zona dolorosa), con resultados preliminares prometedores ${ }^{40}$. De todas formas, se recomienda que ante la duda de si el paciente siente dolor, se trate y se observe la respuesta a esta acción.

\section{MANEJO DEL DOLOR}

Dado la relevancia que tiene el correcto manejo del dolor postoperatorio, con los años se ha utilizado diversas técnicas analgésicas, tales como: sistémica, neuroaxial, bloqueo de nervio periférico y uso de infiltraciones locales, así como también se ha utilizado un variado número de fármacos coad-

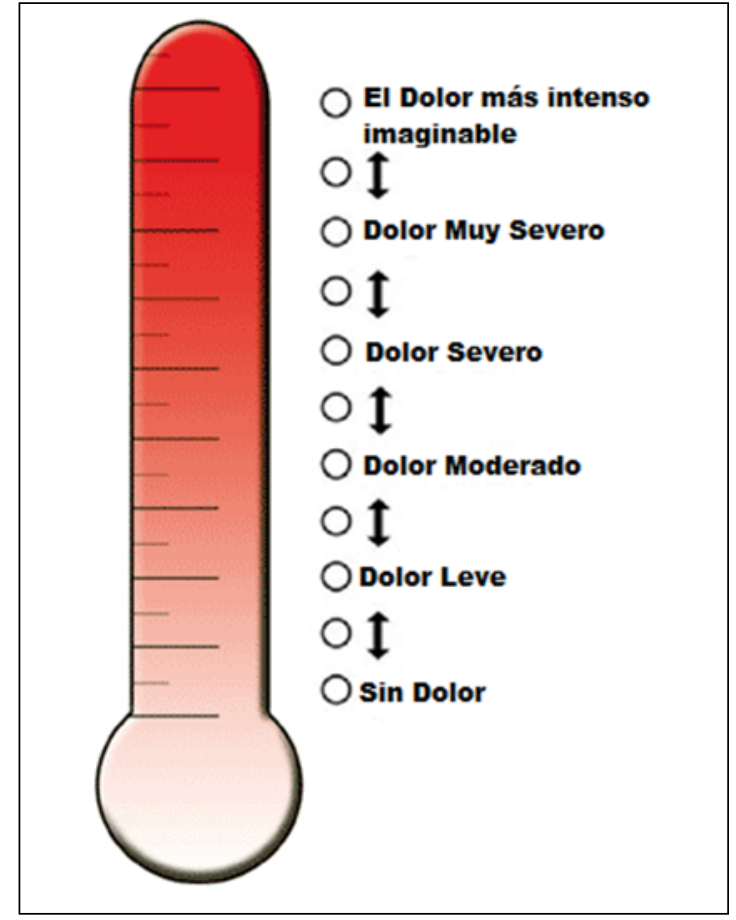

Figura 1. Termómetro del Dolor. Modificado de Herr et $\mathrm{al}^{41}$.

yuvantes. A pesar de esto, ninguna de estas estrategias ha encontrado el resultado perfecto y en el último tiempo se ha comenzado a promover el uso de una combinación de técnicas y agentes analgésicos, para aliviar el dolor en sus diferentes niveles de generación. Esto es lo que se conoce como manejo del dolor "multimodal" 43 . Esta estrategia ha demostrado resultados positivos en variados estudios ya que además de ser al menos igual de efectiva que el uso de una sola técnica o fármaco para el manejo del dolor, se adiciona el beneficio de reducir los efectos adversos comparados con una técnica unimodal ${ }^{44}$. También promueve una recuperación más rápida, una reducción en la estadía hospitalaria, una disminución del consumo de opioides de rescate y un aumento en la participación de programas de rehabilitación precoz, particularmente en pacientes sometidos a artroplastía total de cadera y rodilla $a^{45,46}$.

Por otro lado, el nivel al cual se intenta actuar para manejar el dolor, ha ido cambiando con el tiempo desde un enfoque en el sistema nervioso central al énfasis en el sitio quirúrgico, esto con la intención de disminuir efectos adversos de la analgesia clásica en base a opioides y de otras técnicas regionales clásicas, que si bien es cierto producen buena analgesia pueden asociarse a un retardo en la 
recuperación funcional en cirugía traumatológica. Es así como cada vez ganan más peso las técnicas de bloqueo de nervio periférico y las inyecciones peri o intraarticulares ${ }^{43}$.

A continuación, describiremos elementos de manejo analgésico del AM en las distintas fases del perioperatorio:

\section{Preoperatorio}

Woolf publicó en 1983 resultados de laboratorio que mostraban que la administración de un analgésico antes del estímulo doloroso agudo, era más efectivo para minimizar cambios a nivel de asta dorsal asociados con sensibilización central que la misma analgesia usada luego del estímulo ${ }^{47}$. Sin embargo, sería un error extrapolar estos datos obtenidos experimentalmente a lo que sucede en clínica con los pacientes; es más, dos revisiones sistemáticas al respecto muestran resultados controversiales para el uso de analgesia PRE estímulo versus POST estímulo $^{48,49}$. No obstante parece razonable comenzar con el manejo analgésico lo antes posible, aunque la evidencia al respecto no es categórica en esta conducta.

En el caso de la cirugía traumatológica, un estudio en cirugía de artroplastía de rodilla o cadera, en que se comparó el uso de analgesia con un protocolo estándar en base al uso de analgesia peridural versus un protocolo similar al cual se le agregó el uso de inhibidores de la cicloxigenasa 2 (COX-2), administrados por 2 semanas previo a la cirugía y continuados hasta 10 días postoperatorio, mostró que en este último grupo hubo una disminución significativa de la incidencia de problemas en el control del dolor, definido como EVA mayor a 5 pese a uso de analgésicos postoperatorios ${ }^{50}$.

En otro estudio en que se enrolaron pacientes sometidos a artroplastía total de rodilla, en que al grupo experimental se le administró $50 \mathrm{mg}$ de rofecoxib (COX-2) $24 \mathrm{~h}$ antes y entre 1 a $2 \mathrm{~h}$ previo a la cirugía, $50 \mathrm{mg}$ al día por 5 días postoperatorios y 25 mg al día por 8 días más, comparado con placebo, mostró en el grupo experimental una disminución significativa del uso de opioides intrahospitalarios, menor dolor medido por EVA durante la estadía hospitalaria, menor incidencia de vómitos e importantemente, un significativo aumento del grado de flexión de la rodilla al momento del alta hospitalaria y una semana post alta ${ }^{51}$.

En otro estudio, el uso de gabapentina vía oral dos horas previo a la cirugía de discectomía lumbar, en comparación con placebo, mostró una disminución significativa del dolor postoperatorio y del consumo de fentanilo en las primeras $24 \mathrm{~h}$ del postoperatorio ${ }^{52}$.

Por otro lado, un estudio que comparó el uso de paracetamol endovenoso $30 \mathrm{~min}$ previo a la cirugía, durante la cirugía y previo al cierre de la piel, con un placebo, en pacientes sometidos a cirugía traumatológica de extremidades inferiores, mostró una disminución significativa del dolor postoperatorio en los dos grupos experimentales respecto al placebo $^{53}$.

Por lo tanto, si bien es cierto los estudios mostrados anteriormente no fueron diseñados para la población de AM, parece adecuado en este grupo de pacientes comenzar con el manejo analgésico lo más precoz posible. Sin embargo, no existe consenso en cuál sería la mejor estrategia a utilizar ni tampoco si esto modifica el resultado final de nuestros pacientes.

\section{Intraoperatorio}

\section{Tipo de anestesia para cirugía traumatológica y ortopédica en el AM:}

La pregunta acerca de la superioridad del uso intraoperatorio de una técnica regional de anestesia en comparación a la anestesia general en AM sometidos a cirugía traumatológica, es hasta hoy un punto de controversia.

En general, se puede decir que tanto la anestesia neuroaxial como la anestesia general, han sido utilizadas exitosamente para la cirugía traumatológica por muchos años. Con el tiempo ha existido una tendencia a un aumento del uso de la anestesia regional en la cirugía traumatológica ${ }^{7}$. Entre 1981 y 1982, la anestesia general se ocupaba en el $94,8 \%$ de las cirugías por fractura de cadera en hospitales de Estados Unidos, mientras que entre 1993-1994, se usaba en solo el $49,6 \%{ }^{54}$. La anestesia general tiene las ventajas de poder tener mejor control sobre la duración de la anestesia, de su profundidad y de los parámetros hemodinámicos, además de un control completo sobre la vía aérea y la ventilación del paciente ${ }^{55}$, pero tiene efectos adversos asociados al uso de opioides y específicamente en el adulto mayor, algunos estudios la han asociado a un aumento del riesgo de presentar delirium postoperatorio $^{56}$, sin embargo, no todos los trabajos han demostrado aquello.

El uso de la anestesia neuroaxial cubre las necesidades anestésicas para la mayoría de las cirugías traumatológicas, añadiendo la simplicidad de la técnica, prolongación del efecto analgésico en el postoperatorio, y disminución de algunos efectos adversos, como son las náuseas y los vómitos entre 
otros. Sin embargo, presenta algunas posibles complicaciones como la hipotensión intraoperatoria, bloqueo regional inadecuado, retención urinaria y complicaciones muy inhabituales como cefalea post punción meníngea, hematoma epidural o infección ${ }^{57,58}$.

Macfarlane en una revisión sistemática que buscó diferentes resultados comparando el uso de anestesia regional con anestesia general en cirugía de cadera, encontró en el grupo de anestesia regional una disminución del dolor postoperatorio, en el uso de opioides y de náuseas y vómitos postoperatorios ${ }^{59}$.

En los últimos años ha crecido el interés por el uso de bloqueos de nervio periférico en las cirugías traumatológicas, tanto para dar anestesia durante la cirugía, como también para el manejo del dolor postoperatorio ${ }^{36}$. Una revisión en la que se incluyeron 17 estudios randomizados, con un total de 888 pacientes sometidos a cirugía por fractura de cadera, con 9 estudios en los que se usó bloqueo de nervio periférico previo a la cirugía y 8 en forma perioperatoria, mostró una reducción significativa del dolor postoperatorio y del uso de analgésicos durante la cirugía y en el postoperatorio ${ }^{60}$.

Por lo tanto, la evidencia disponible señala que la anestesia regional como técnica anestésica en cirugía traumatológica, especialmente para cirugía de cadera (en su gran mayoría pacientes adultos mayores), puede disminuir el dolor, las náuseas y vómitos y el uso de opioides postoperatorios.

\section{Uso de inyección periarticular intraoperatoria para el manejo del dolor}

Hace algunos años se ideó la inyección de analgésicos en el sitio operatorio, con el fin de evitar la sensibilización periférica y central y así disminuir el dolor postoperatorio y hacerlo de más fácil manejo.

Un estudio en el que se comparó la utilización de una inyección periarticular de ropivacaína, ketorolaco, morfina y epinefrina versus placebo durante el intraoperatorio en artroplastía total de rodilla, mostró una reducción significativa de los requerimientos de analgesia controlada por el paciente (PCA) y una mejora en la satisfacción del paciente $^{61}$.

Luego, con el fin de estandarizar una técnica, Kerr y Kohan, el año 2008, crearon la analgesia por infiltración local (LIA, por sus siglas en inglés). En un estudio con 325 pacientes sometidos a artroplastía de rodilla o cadera, utilizaron inyecciones locales en el sitio operatorio con una mezcla de drogas (ropivacaína, ketorolaco y epinefrina), encontrando un control satisfactorio del dolor por parte del paciente, una movilización y alta precoz con ausencia de complicaciones o efectos adversos importantes $^{62}$.

Posteriormente, esta técnica ha ganado adeptos y han sido publicados estudios de mayor calidad metodológica. Un estudio, en que se incluyó pacientes sometidos a artroplastía total bilateral de rodilla, mostró una disminución significativa del dolor postoperatorio en reposo y con la movilización a las $32 \mathrm{~h}$, al compararlo con placebo ${ }^{63}$. Sin embargo, los mismos autores no lograron demostrar un beneficio en el uso de LIA en artroplastía total de cadera bilateral ${ }^{64}$. En otro estudio en artroplastía total de rodilla, se mostró una disminución del dolor y del uso de morfina postoperatoria, con un tiempo menor de estadía hospitalaria y una mejor satisfacción del paciente, al comparar LIA con placebo ${ }^{65}$. Por otra parte, una revisión sistemática, concluyó que la evidencia a favor del uso de la LIA en artroplastía de cadera proviene de un número pequeño de ensayos clínicos, mostrando ser superior que placebo y en un estudio, superior a analgesia epidural, pero no ha mostrado beneficios adicionales al ser utilizada en el contexto del uso de analgesia multimodal ${ }^{66}$. En otra revisión sistemática con metanálisis, que compara LIA en artroplastía de cadera con inyección o no inyección de placebo, encontró una fuerte evidencia en relación al uso de LIA y la reducción del dolor postoperatorio en reposo a las 4 y $24 \mathrm{~h}$ y al dolor postoperatorio dinámico a las 4 y $6 \mathrm{~h}$, y una reducción del consumo de analgésicos entre las 0 y $24 \mathrm{~h}$, sin aumento en el número de efectos adversos ${ }^{67}$.

Por lo tanto, la LIA parece ser una técnica adecuada para reducir el dolor postoperatorio y el consumo de analgésicos luego de artroplastía de rodilla o cadera, por ser fácil de implementar y en estudios preliminares, libre de efectos adversos importantes. Sin embargo, es una técnica relativamente nueva y hace falta más evidencia para recomendar su uso generalizado. Extrapolar su uso para cirugía en la cual existe preservación de la articulación puede ser controvertido, dado que las inyecciones intraarticulares de anestésicos locales se han relacionado con un posible daño de cartílagos.

\section{Postoperatorio}

\section{Analgesia sistémica}

La analgesia sistémica postoperatoria se debe utilizar en forma escalonada y en función de los requerimientos del paciente. Los AINEs, de no existir contraindicaciones, se recomiendan para el manejo del dolor postoperatorio, pues disminuyen el dolor y el consumo de analgésicos adicionales. Se reco- 
mienda su uso asociado a opioides fuertes, como morfina o fentanilo, para el dolor de alta intensidad y a opioides débiles, como tramadol, en caso de dolor de baja intensidad. Al mismo tiempo se recomienda que los opioides sean combinados con analgésicos no opioides con el fin de reducir sus dosis y los efectos adversos asociados. El uso de paracetamol se recomienda solo en combinación con otros analgésicos, como tratamiento de base para cualquier intensidad de dolor, ya que puede disminuir los requerimientos adicionales de drogas ${ }^{68,69}$.

Una revisión sistemática, muestra que no existe la evidencia adecuada, dado un importante número de estudios sin la validez interna y externa necesaria, para la toma de decisiones en la práctica clínica o para la elaboración de guías terapéuticas acerca del uso de drogas analgésicas para el manejo del dolor postoperatorio luego de cirugía traumatológica u ortopédica ${ }^{70}$.

\section{Analgesia regional}

Los bloqueos de nervio periférico se han convertido en el último tiempo en la técnica analgésica más recomendada para cirugía ortopédica mayor de miembro inferior, siendo propuesta como una alternativa eficaz frente a la analgesia peridural o la PCA endovenosa para el control del dolor postoperatorio ${ }^{7,71}$. El bloqueo periférico de nervio femoral es recomendado por algunos incluso por sobre técnicas neuroaxiales y endovenosas, considerando la disminución de efectos adversos para la cirugía ortopédica mayor de extremidades inferiores $^{72}$.

En el caso de la artroplastía total de cadera, la analgesia postoperatoria puede lograrse eficazmente con un catéter peridural, como también con un bloqueo de plexo lumbar; ambas técnicas disminuyen la necesidad de opiáceos en el postoperatorio $^{73}$. Tanto la analgesia peridural, como el bloqueo continuo de nervio femoral, son más efectivos que el uso de PCA endovenosa en pacientes sometidos a artroplastía total de cadera ${ }^{7}$. El bloqueo de plexo lumbar es más eficaz que el bloqueo de nervio femoral para la artroplastía total de cadera, pero con complicaciones potencialmente más graves, por lo que el riesgo/beneficio debe considerarse en forma individual al elegir un bloqueo por sobre el otro ${ }^{69}$.

Por otro lado, para la artroplastía total de rodilla, tanto la técnica peridural continua como el bloqueo femoral controlan eficazmente el dolor postoperatorio a la vez que facilitan la rehabilitación $^{74,75}$, siendo más efectivos que la analgesia endovenosa ${ }^{76}$. El beneficio de un bloqueo femoral continuo frente al uso de una sola inyección, no es claro, aunque algunos estudios lo catalogan como mejor para reducir el dolor postoperatorio, pero sin diferencias en la recuperación funcional. De todas formas no hay recomendaciones específicas sobre cual de los dos métodos preferir.

Es importante señalar la importancia de tener un protocolo claro de prevención del riesgo de caídas secundario al bloqueo muscular del cuádriceps asociado al bloqueo femoral, considerando que se le agrega un riesgo adicional a una población que tiene más riesgo no solo de caídas, sino de fracturas asociadas a estas.

\section{Comparación entre distintas técnicas para la analgesia postoperatoria}

En una revisión sistemática del año 2011 de buena calidad metodológica, que buscó comparar la efectividad de distintas modalidades para el manejo del dolor postoperatorio para la cirugía de fractura de cadera, que incluyó 83 estudios (64 revisiones sistemáticas) sobre el uso de bloqueo de nervio periférico, analgesia espinal, analgesia sistémica, tracción, manejo multimodal, neuroestimulación, rehabilitación y medicina alternativa y complementaria, se concluyó que el uso de bloqueos de nervio disminuye efectivamente el dolor postoperatorio al compararlos con no utilizarlos, con resultados estadísticamente significativos para la analgesia peridural, el bloqueo de nervio femoral, el bloqueo de plexo lumbar y la combinación de bloqueos de nervio y no significativos en el caso del bloqueo femoral 3 en 1 y de los bloqueos de fascia ilíaca. No se pudo sacar conclusiones acerca del resto de intervenciones analizadas, dado que existe evidencia insuficiente acerca de sus beneficios y efectos adversos. En cuanto a otros resultados, solo hubo disminución significativa en la incidencia de delirium al comparar el uso de algún bloqueo de nervio frente a no usarlo. No hubo diferencias significativas al comparar bloqueo de nervio con anestesia neuroaxial en cuanto a dolor postoperatorio, uso adicional de analgésicos o delirium ${ }^{77}$.

\section{CONCLUSIONES}

La población a nivel mundial y nacional está envejeciendo, por lo tanto cada vez más nos vamos a ver enfrentados a este tipo de pacientes. Dentro de los procedimientos a los que van a ser sometidos, la cirugía traumatológica y ortopédica es de las más frecuentes.

El conocer la fisiología del envejecimiento, los cambios farmacológicos y las particularidades en la evaluación y manejo del dolor perioperatorio en 
los adultos mayores, es fundamental para tener un adecuado enfrentamiento hacia estos pacientes.

El manejo analgésico del AM sometido a una cirugía traumatológica debe ser considerado como un continuo en todas las fases del perioperatorio, enfrentando a cada paciente de forma personalizada con un enfoque multimodal.
La gran mayoría de la literatura publicada no está diseñada específicamente para resolver las interrogantes en este grupo de pacientes, por lo que es difícil extrapolar o generalizar los datos publicados a la población de AM, sin embargo, es la mejor evidencia disponible actualmente y debemos entenderla en esos términos.

\section{REFERENCIAS}

1. United Nations, Department of Economic and Social Affairs, Population Division (2013). World Population Prospects: The 2012 Revision, Volume I: Comprehensive Tables ST/ESA/ SER.A/336.

2. Instituto Nacional de Estadísticas, Departamento de Demografía: Programa de Proyecciones de la Población: CHILE: Proyecciones y Estimaciones de Población por sexo y edad. 1990 - 2020. 2003.

3. Hadzic A. Anestesia regional en ancianos. En: Tratado de Anestesia Regional y Manejo del Dolor Agudo. 1st ed. México: Mc GrawHill, 2010: 787-798.

4. Clergue F, Auroy Y, Pequignot F, Jougla E, Lienhart A, Laxenaire MC. French survey of anesthesia in 1996. Anesthesiology 1999; 91 : 1509-1520.

5. Kadono Y, Yasunaga H, Horiguchi $\mathrm{H}$, et al. Statistics for orthopedic surgery 2006-2007: data from the Japanese Diagnosis Procedure Combination database. Journal of orthopaedic science: official journal of the Japanese Orthopaedic Association 2010; 15: 162-70 doi: 10.1007/s00776-009-1448-2.

6. Bready LL, Mullins RM, Noorily SH, Smith RB. Acute and Postoperative. En: Decision Making in Anesthesiology: An algorithm approach. 3rd ed. St. Louis: Mosby, 2000; 594: 95.

7. Silverstein JH, Rooke GA, Reves JG, McLeskey ChH. Total Hip Replacement, Joint Replacement, and Hip Fracture. En: Geriatric Anesthesiology. 2nd ed. New York: Springer, 2008: 355-367.

8. Zuckerman JD. Hip fracture. N
Engl J Med. 1996; 334: 1519-1525.

9. Macyntire PE, Walker SM, Rowbotham DJ. Acute Pain Management in the Elderly Patient. Clinical Pain Management: Acute Pain. 2nd edition. London: Hodder Arnold, 2008. Tabla 28.1. p 506.

10. Miller RD. Anestesia en ancianos. En: Miller Anestesia. Trans. Francico López. 6th ed. Génova: Elsevier España, 2005: 2435-2449.

11. Aubrun F. Management of postoperative analgesia in elderly patients. Reg Anesth Pain Med. 2005; 30: 363-379.

12. Silverstein JH, Rooke GA, Reves JG, McLeskey ChH. Alteration in Metabolic Functions and Electrolytes. En: Geriatric Anesthesiology. 2nd ed. New York: Springer, 2008: 97-107.

13. Priebe HJ. The aged cardiovascular risk patient. Br J Anaesth. 2000; 85: 763-778.

14. Halaszynski T. Influences of the aging process on acute perioperative pain management in elderly and cognitively impaired patients. The Ochsner journal 2013; 13: 228-247.

15. Sprung J, Gajic O, Warner DO. Review article: Age related alterations in respiratory function - Anesthetic considerations. Can J Anaesth. 2006; 53: 1244-1257.

16. Sinatra RS, de Leon Cassasola OA, Ginsberg B, Viscusi ER. Elderly High Risk and Cognitively Impaired Patients. En: Acute Pain Management. 1st ed. New York: Cambridge University Press, 2009: 527-529.

17. Vestal RE, Wood AJ, Shand DG. Reduced beta-adrenoceptor sensitivity in the elderly. Clin Pharmacol Ther. 1979; 26(2): 181186.
18. Sessler DI. Temperature monitoring and perioperative thermoregulation. Anesthesiology 2008; 109(2): 318338.

19. Shorten G. Postoperative Pain Management in the Elderly. En: Postoperative Pain Management: An Evidence-Based Guide to Practice. 1st ed. Philadelphia: Saunders-Elsevier, 2006: 219-224.

20. Veering BT, Burm AG, van Kleef JW, Hennis PJ, Spierdijk

J. Epidural anesthesia with bupivacaine: effects of age on neural blockade and pharmacokinetics. Anesth Analg. 1987; 66: 589-593.

21. Bromage PR. Exaggerated spread of epidural analgesia in arteriosclerotic patients. Dosage in relation to biological and chronological ageing. Br Med J. 1962; 2: 1634-1638.

22. Fernández-Galinski D, Rue M, Moral V, Castells C, Puig MM. Spinal anesthesia with bupivacaine and fentanyl in geriatric patients. Anesth Analg. 1996; 83: 537-541.

23. Shantha TR, Evans JA. The relationship of epidural anesthesia to neural membranes and arachnoid villi. Anesthesiology 1972; 37: 543557.

24. Rathmell JP, Pino CA, Taylor R, Patrin T, Viani BA. Intrathecal morphine for postoperative analgesia: a randomized, controlled, dose-ranging study after hip and knee arthroplasty. Anesth Analg. 2003; 97: 1452-1457.

25. Veering BT, Burm AG, van Kleef JW, Hennis PJ, Spierdijk J. Spinal anesthesia with glucosefree bupivacaine: effects of age on neural blockade and pharmacokinetics. Anesth Analg. 1987; 66: 965-970. 
26. Veering BT, Burm AG, Spierdijk J. Spinal anaesthesia with hyperbaric bupivacaine. Effects of age on neural blockade and pharmacokinetics. Br J Anaesth. 1988; 60: 187-194.

27. Horlocker TT, Wedel DJ, Rowlingson JC, et al. Regional anesthesia in the patient receiving antithrombotic or thrombolytic therapy: American Society of Regional Anesthesia and Pain Medicine Evidence-Based Guidelines (Third Edition). Reg Anesth Pain Med. 2010; 35: 64101.

28. Silverstein JH, Rooke GA, Reves JG, McLeskey ChH. Anesthesia Considerations for Geriatric Outpatients. En: Geriatric Anesthesiology. 2nd ed. New York: Springer, 2008: 308-321.

29. Miller RD. Dolor agudo postoperatorio. En: Miller Anestesia. Trans. Francisco López. 6th ed. Génova: Elsevier España, 2005: 2729-2762.

30. Zywiel MG, Prabhu A, Perruccio AV, Gandhi R. The influence of anesthesia and pain management on cognitive dysfunction after joint arthroplasty: a systematic review. Clin Orthop Relat Res. 2014; 472: 1453-1466.

31. Macintyre PE, Scott DA, Schug SA et al. The older patient. En: Acute Pain Management: Scientific Evidence. 3rd ed. Melbourne: ANZCA \& FPM, 2010: 400-410.

32. Gibson SJ. IASP global year against pain in older persons: highlighting the current status and future perspectives in geriatric pain. Expert Rev Neurother. 2007; 7: 627-635 doi: 10.1586/14737175.7.6.627.

33. Small SA. Age-related memory decline: current concepts and future directions. Arch Neurol. 2001; 58: 360-364.

34. Warfield CA, Bajwa ZH. Pain in the elderly. En: Principles \& Practice of Pain Medicine. 2nd ed. New York: McGraw-Hill, 2004; 571-579.

35. Farrell MJ, Katz B, Helme RD. The impact of dementia on the pain experience. Pain 1996; 67: 7-15.
36. Silverstein JH, Rooke GA, Reves JG, McLeskey ChH. Pain Management. En: Geriatric Anesthesiology. 2nd ed. New York: Springer, 2008; 308-321.

37. Morrison RS, Siu AL. A comparison of pain and its treatment in advanced dementia and cognitively intact patients with hip fracture. J Pain Symptom Manage. 2000; 19: 240-248.

38. Banos JE, Bosch F, Canellas M, Bassols A, Ortega F, Bigorra J. Acceptability of visual analogue scales in the clinical setting: a comparison with verbal rating scales in postoperative pain. Methods Find Exp Clin Pharmacol. 1989; 11: 123-127.

39. Macintyre PE, Scott DA, Schug SA et al. Assesment and Measurement of Pain and its Treatment. En: Acute Pain Management Scientific Evidence. 3rd ed. Melbourne: ANZCA \& FPM, 2010; 35-45.

40. Rakel B, Herr K. Assessment and treatment of postoperative pain in older adults. J Perianesth Nurs. 2004; 19: 194-208.

41. Herr K, Spratt KF, Garand L, Li L. Evaluation of the Iowa pain thermometer and other selected pain intensity scales in younger and older adult cohorts using controlled clinical pain: a preliminary study. Pain Med. 2007; 8: 585-600.

42. Herr KA, Spratt K, Mobily PR, Richardson G. Pain intensity assessment in older adults: use of experimental pain to compare psychometric properties and usability of selected pain scales with younger adults. Clin J Pain 2004; 20: 207-219.

43. Memtsoudis SG. Guest editorial: Perioperative pain management in orthopaedic surgery: editorial comment. Clin Orthop Relat Res. 2014; 472: 1375-1376.

44. Duellman TJ, Gaffigan C, Milbrandt JC, Allan DG. Multimodal, pre-emptive analgesia decreases the length of hospital stay following total joint arthroplasty. Orthopedics 2009; 32: 167.

45. Kehlet H, Wilmore DW. Multimodal strategies to improve surgical outcome. Am J Surg 2002;
183: 630-641.

46. Jin F, Chung F. Multimodal analgesia for postoperative pain control. J Clin Anesth. 2001; 13: 524-539.

47. Woolf CJ. Evidence for a central component of post-injury pain hypersensitivity. Nature 1983; 306: 686-688.

48. Dahl JB, Moiniche S. Pre-emptive analgesia. Br Med Bull. 2004; 71 : 13-27.

49. Moiniche S, Kehlet H, Dahl JB. A qualitative and quantitative systematic review of preemptive analgesia for postoperative pain relief: the role of timing of analgesia. Anesthesiology 2002; 96: 725-741.

50. Mallory TH, Lombardi AV, Jr., Fada RA, Dodds KL, Adams JB. Pain management for joint arthroplasty: preemptive analgesia. J Arthroplasty 2002; 17(Suppl 1): 129-133.

51. Buvanendran A, Kroin JS, Tuman $\mathrm{KJ}$, et al. Effects of perioperative administration of a selective cyclooxygenase 2 inhibitor on pain management and recovery of function after knee replacement: a randomized controlled trial. JAMA 2003; 290: 2411-2418.

52. Pandey CK, Sahay S, Gupta D, et al. Preemptive gabapentin decreases postoperative pain after lumbar discoidectomy. Can J Anaesth. 2004; 51: 986-989.

53. Khalili G, Janghorbani M, Saryazdi H, Emaminejad A. Effect of preemptive and preventive acetaminophen on postoperative pain score: a randomized, doubleblind trial of patients undergoing lower extremity surgery. J Clin Anesth. 2013; 25: 188-192.

54. O'Hara DA, Duff A, Berlin JA, et al. The effect of anesthetic technique on postoperative outcomes in hip fracture repair. Anesthesiology 2000; 92: 947-957.

55. Semple T, Toh GW. Anaesthesia and hip fracture: a review of the current literature. 2007. Accesado el 28 de octubre de 2014 en: http:// www.anzca.edu.au/resources/ college-publications/pdfs/booksand-publications/Australasian $\% 20$ 
Anaesthesia/australasiananaesthesia-2007/Semple.pdf

56. Beaupre LA, Jones CA, Saunders LD, Johnston DW, Buckingham J, Majumdar SR. Best practices for elderly hip fracture patients. A systematic overview of the evidence. J Gen Intern Med. 2005; 20: 1019-1025.

57. Longo S. Regional versus general anesthesia. Curr Opin Anaesthesiol. 2000; 13: 539-543.

58. Singelyn FJ, Ferrant T, Malisse MF, Joris D. Effects of intravenous patient-controlled analgesia with morphine, continuous epidural analgesia, and continuous femoral nerve sheath block on rehabilitation after unilateral total-hip arthroplasty. Reg Anesth Pain Med. 2005; 30: 452-457.

59. Macfarlane AJ, Prasad GA, Chan VW, Brull R. Does regional anaesthesia improve outcome after total hip arthroplasty? A systematic review. Br J Anaesth. 2009; 103: 335-345.

60. Parker MJ, Griffiths R, Appadu BN. Nerve blocks (subcostal, lateral cutaneous, femoral, triple, psoas) for hip fractures. Cochrane Database Syst Rev. 2002(1): CD001159.

61. Busch CA, Shore BJ, Bhandari $R$, et al. Efficacy of periarticular multimodal drug injection in total knee arthroplasty. A randomized trial. J Bone Joint Surg Am. 2006; 88: 959-963.

62. Kerr DR, Kohan L. Local infiltration analgesia: a technique for the control of acute postoperative pain following knee and hip surgery: a case study of 325 patients. Acta Orthop. 2008; 79: 174-183.

63. Andersen LO, Husted H, Otte KS, Kristensen BB, Kehlet H. High-volume infiltration analgesia in total knee arthroplasty: a randomized, double-blind, placebocontrolled trial. Acta Anaesthesiol Scand. 2008; 52: 1331-1335.

64. Andersen LO, Otte KS, Husted H, Gaarn-Larsen L, Kristensen B, Kehlet H. High-volume infiltration analgesia in bilateral hip arthroplasty. A randomized, doubleblind placebo-controlled trial. Acta Orthop. 2011; 82: 423-426.

65. Essving P, Axelsson K, Kjellberg J, Wallgren O, Gupta A, Lundin A. Reduced morphine consumption and pain intensity with local infiltration analgesia (LIA) following total knee arthroplasty. Acta Orthop. 2010; 81: 354-360.

66. McCarthy D, Iohom G. Local Infiltration Analgesia for Postoperative Pain Control following Total Hip Arthroplasty: A Systematic Review. Anesthesiology Research and Practice 2012; 2012: 709531.

67. Yin JB, Cui GB, Mi MS, et al. Local Infiltration Analgesia for Postoperative Pain After Hip Arthroplasty: A Systematic Review and Meta-Analysis. J Pain 2014;15: 781-799.

68. Fischer HB, Simanski CJ, Sharp $\mathrm{C}$, et al. A procedure-specific systematic review and consensus recommendations for postoperative analgesia following total knee arthroplasty. Anaesthesia 2008; 63: 1105-1123.

69. Fischer HB, Simanski CJ. A procedure-specific systematic review and consensus recommendations for analgesia after total hip replacement. Anaesthesia 2005; 60: 1189-1202.

70. Montane E, Vallano A, Aguilera C, Vidal X, Laporte JR. Analgesics for pain after traumatic or orthopaedic surgery: what is the evidence-a systematic review. Eur J Clin Pharmacol. 2006; 62: 971-988.

71. Singelyn F, Gouverneur JM.
Postoperative analgesia after total hip arthroplasty: IV PCA with morphine, patient-controlled epidural analgesia, or continous "3-in-1 block"; a prospective evaluation by our acute pain service in moe than 1300 patients. J Clin Anesth. 1999; 11: 550-554.

72. Sinatra RS, Torres J, Bustos AM. Pain management after major orthopaedic surgery. Current strategies and new concepts. J Am Acad Orthop Surg 2002; 10: 117129.

73. Stevens RD, Van Gessel E, Flory N, Fournier R, Gamulin Z. Lumbar plexus block reduces pain and blood loss associated with total hip arthroplasty. Anesthesiology 2000; 93: 115-121.

74. Capdevila X, Barthelet Y, Biboulet P, Ryckwaert Y, Rubenovitch J, d'Athis F. Effects of perioperative analgesic technique on the surgical outcome and duration of rehabilitation after major knee surgery. Anesthesiology 1999; 91: 8-15.

75. Ganapathy S, Wasserman RA, Watson JT, et al. Modified continuous femoral three-in-one block for postoperative pain after total knee arthroplasty. Anesth Analg. 1999; 89: 1197-1202.

76. Singelyn FJ, Deyaert M, Joris D, Pendeville E, Gouverneur JM. Effects of intravenous patientcontrolled analgesia with morphine, continuous epidural analgesia, and continuous three-in-one block on postoperative pain and knee rehabilitation after unilateral total knee arthroplasty. Anesth Analg. 1998; 87: 88-92.

77. Abou-Setta AM, Beaupre LA, Rashiq S, et al. Comparative effectiveness of pain management interventions for hip fracture: a systematic review. Ann Int Med. 2011; 155: 234-245.

\section{Correspondencia a: \\ Dr. Pablo Miranda H. \\ pfmirand@uc.cl}

